\title{
Perturbation in Nonlinear Operator Dynamical Systems
}

\author{
Reza Ahangar \\ 700 University BLVD, MSC 172, Department of Mathematics, Texas A \& M University-Kingsville Kingsville, TX 78363, USA
}

Copyright (C) 2019 by authors, all rights reserved. Authors agree that this article remains permanently open access under the terms of the Creative Commons Attribution License 4.0 International License

\begin{abstract}
An introduction to nonlinear operator dynamical systems with a brief technical notation will be presented. The operator is designed to include all nonanticipating (causal) delay and functional dierential equations. Condi- tions for the existence and uniqueness of these noanticipating operator dierential equations (NODE) will be investigated. At last the perturbed solutions of these type nonlinear systems and some properties will be presented.
\end{abstract}

Keywords Nonlinear Dynamical Systems, Lipschitzian Operators, k-norm, Nonanticipating, Initial Domain, Perturbation

\section{Introduction}

Applications of functional and operator differential equations were studied extensively during the past decades, particularly solutions to automatic control problems using nonlinear operator systems. Delay and Functional Differential Equations were studied by Hale J. 1976, Driver 1977. The stability and asymptotic behavior of nonlinear systems of delay and functional differential equations in abstract space have been studied by Lakshmikantham 1981 and Hale 1988. The monographs of Zemanian 1968 and Tutschchke 1988 are good resources to study the beginning of generalized dynamical system on both partial and ordinary differential equations.

Integrodifferential equations and the systems involving semigroup of operators have been investigated extensively by many authors during the past decades. We can see the history of evolution and development of nonlinear studies through nonlinear variation of parameters in Lakshmikantham 1998. For further references of earlier developments see Kisynski 1976 and Kerin 1983.

The applications of operator approach on the study of control, optimal control, and automatic control problems are also divided on the selection of the type of operators involved in the dynamical systems and whether the model is deterministic or stochastic. Most of these works did not go beyond the semigroup or integral operators.

The above mentioned dynamical systems which involved delay, integral, and functional differential equations involved linear operators and have not been studied in a unified approach. The study of nonlinear operator differential equations has undergone intensive development particularly in integral equations and nonlinear equations of evolution and boundary value problems for partial differential equations (see Vainberg1973).

The first systematic unified approach of generalized dynamical systems, when the operator is of an exponential type was used by Bogdan (1981 and 1982) to study the automatic control for spaceship navigation.

We were looking for a system of nonlinear operators that the future path will be determined only by our knowledge from the past up to the present. Also we want to develop a deterministic form of a nonanticipating operator that can be extended to a stochastic system by selecting a suitable probabilistic measurable space. The generalized dynamical systems satisfying nonanticipating operator differential equations are applied to optimal control problems (Ahangar 1986 and 1989).

Unfortunately, a unified approach toward the solution of the nonlinear operator optimal control problems of the generalized dynamical systems has not been investgated and the objective of this paper is to present this approach to study the optimal automatic control problems with all varieties of nonlinear operator differential equations.

Generalized dynamical systems, when the operator is of an exponential type, have been studied by Bogdan 1981 and 1982. The nonanticipating dynamical model and optimal control and its application to automatic control has been studied (see Ahangar et al). 
By Generalized Dynamical Systems, we mean a nonlinear operator differential equation

$$
y^{\prime}(t)=f(t, y(t), T(y)(t)) \quad \text { fort }>t_{0}
$$

and $y(t)=\phi(t)$ for all $t \leq t_{0}$. This dynamical system could be interpreted and applied to a certain operator satisfying the conditions for the solution. We will study the generalized dynamical systems with nonanticipating and Lipschitzian operator. This model could be used for all operators of functional, delay, and integral differential equations. It is actually a unifying methods for all research problems done on these categories.

\section{Nonlinear Operator Differential Equations}

Basic Definitions: In what follows $Y, Z$, and $U$ will be Banach Spaces, and $I=[O, a], S=\{t: t<O\}$, and $J=I \cup S$ for an arbitrary but fixed real number $a$. Denote by $M(K, Y)$ the Banach space of all essentially bounded Bochner measurable functions from an interval $K$ into a Banach space $Y$ with respect to classical Lebesgue measure. The norm in this space will be the essential supremum norm $\|.\| \infty$.

Define $\operatorname{Lip}(K, Y)$ to be the Banach space of all Lipschitzian functions $y: K \rightarrow Y$ strongly differentiable almost everywhere on $K$. For a fixed initial function $\phi \in L(S, Y)$ we define the Initial Domain, denoted by $D(\phi, Y)$, to be the subset of $L(S, Y)$ consisting of all functions $y$ such that $y(t)=\phi(t)$ for all $t \in S$. That is, $\quad D(\phi, Y)=\left\{y \in L(J, Y):\left.y\right|_{s}=\phi\right\}$.

We denote by $D(\phi, \omega, Y)$ a subset of the Lipschitzian Initial Domain of all trajectory with a particular Lipschitz constant $\omega$. These initial domains can be considered a subset of the space $M(I, Y)$. Lipschitzian Space (or simply Lip-Space), denoted by $\operatorname{Lip}(K, Y ; Z)$, is the set of all functions $f: K \times Y \rightarrow Z$ such that $f(t, y)$ is uniformly bounded, Lipschitzian in $y$, and measurable in $t$.

Lipschitzian Operator: The infimum of all Lipschitzian constants $L$ will be denoted by $\|f\|$. An operator $P$ from a subset $D$ of $Y$ into $Z$ is said to be Lipschitzian if there exists a constant $b$ such that

$$
\left|P\left(y_{1}\right)-P\left(y_{2}\right)\right| \leq b\left|y_{1}-y_{2}\right|
$$

for every $y_{1}, y_{2} \in Y$. The infimum of the value of $\mathrm{b}$ in (2.1) is denoted by $\|P\|$.

On the space $\mathrm{M}(\mathrm{I}, \mathrm{Y})$, we shall introduce a family of norms, called k-norms by the formula

$$
\|y\|_{k}=e s s . \sup \left\{e^{-k t}|y(t)|: t \in I\right\}<\infty,
$$

for any fixed real number $\mathrm{k}$.

Exponential Type Operator: From this definition follows the inequality

$$
|y(t)| \leq\|y\|_{k} e^{k t} \quad \text { for almost all } t \in I
$$

$|y(t)| \leq\|y\|_{k} e^{k t}$ for almost all $\mathrm{t}$ in I. Notice that for every $\mathrm{k}$, the $\mathrm{k}$ norms \|\|$_{k}$ and $\|$. $\|_{0}$ are equivalent. A Lipschitzian operator $\mathrm{P}$ from a subset domain $\mathrm{D} \subset \mathrm{M}(\mathrm{J}, \mathrm{Y})$ into the space $\mathrm{M}(\mathrm{I}, \mathrm{U})$ is called an operator of exponential type, if for some constants $\mathrm{b}$ and $k_{0}$,

$$
\left.\|P(y)-P(z)\|_{k} \leq b \| y-z\right) \|_{k}
$$

for all $\mathrm{y}$ and $\mathrm{z}$ in the domain $\mathrm{D}$ and all $k \geq k_{0}$.

Induced Operator:

We define the k-norm of the exponential type by:

$$
\|P\|_{k}=\inf \{b: b \text { satisfies in (1.3) }\}
$$

Consider a fixed function $f \in \operatorname{Lip}(I, Y ; Z)$. Define an operator $F: D \subset M(I, Y) \rightarrow M(I, Z)$ such as

$$
z=F(y) \quad \text { if and only if } \quad z(t)=f(t, y(t), T(y)(t))
$$

This is unique way to define and operator and it is called the induced operator generated by $f$. Symbolically means that the induced operator $\mathrm{F}$ maps a function $\mathrm{y}$ to $\mathrm{z}$ through a transformation given by a nonlinear transformation $\mathrm{f}$.

Properties of the nonlinear operator $F$ induced by the function $f$ have been studied in Bogdan 'et al' (1981). In particular, we know that, for $f \in \operatorname{Lip}(I, Y ; Z)$ and $y \in M(I, Y)$, the function $F: I \rightarrow Z$ defined by $F(t)=f(t, y(t))$ belongs to the space of measurable functions $M(I, Z)$. 
Nonanticipating Operator: An operator $T: D(\phi, Y) \rightarrow M(I, Z)$ is called nonanticipating if, for every two functions $y, z \in D(\phi, Y)$ and every $s \in I$, the fact that $y(t)=z(t)$ for almost all $t<s$ implies that $T(y)(t)=T(z)(t)$ for almost all $t<s$.

When an operator $T$ is nonanticipating, the future values of the input will have no effect on the present state. One can prove that the delay, integral operators, the composition and Cartesian product of nonanticipating and Lipschitzian operators are Nonanticipating and Lipschitzian. Furthermore, the operator $F$ induced by the function $f$ is a well defined, nonanticipating, and Lipschitzian operator.

Let us assume $\mathrm{h}=\mathrm{F}(\mathrm{y})$ operator defined in (1.4). Assume $g_{0}$ denote the function $f(t, 0, T(0))$. Now from the estimate

$$
|h(t)|=\mid f(t, y(t), T(y)(t)|=| f(t, y(t), z(t)) \mid
$$

where $z=T(y)$ and

$$
\begin{gathered}
|h(t)| \leq \mid f(t, y(t), z(t))-f(t, 0, T(\phi)(0)|+| f(t, 0, T(0) \mid \\
\leq\|f\||y(t)|+\left|f\left(t, 0, z_{0}\right)\right| \leq\|f\||y(t)|+\|f\| \\
\quad \leq\|f\|(|y(t)|+1) \\
|h(t)|=\mid f(t, y(t), T(y)(t) \mid \leq\|f\|(|y(t)|+1)
\end{gathered}
$$

follows that for almost all $t \in K$ (an arbitrary interval) we have

$$
|h(t)| \leq L\|y\|_{0}+\|g\|_{0} .
$$

The last inequality proves that the function $\mathrm{z}$ is essentially bounded and thus it belongs to the space $M(K, Z)$.

\section{Solution to the Nonlinear Operator Differential Equation}

One can prove that variable delay, constant delay, integral, composition, and Cartesian products of nonanticipating operators are nonanticipating operators.

Let us define the operator $H$ from $M(I, Y)$ into $D(\phi, Y)$ by the following relations

$$
y=H(z) \Leftrightarrow y(t)=\left\{\begin{array}{c}
\phi(t) \text { for } t \leq 0 \\
\phi(0)+\int_{[0, t]} z(s) d s
\end{array}\right.
$$

for all $t \in I$. This operator is equivalent to:

$$
\begin{aligned}
y^{\prime}(t) & =z(t) \text { for } t>0 \\
y(t) & =\phi(t) \text { fot } \mathrm{t} \leq 0
\end{aligned}
$$

In the sequel we will need the following lemmas.

Lemma 3.1: The operator $\mathrm{H}$ as defined by the formula (2.5.1) satisfies the following estimate

$$
\|H\|_{k} \leq \frac{1}{k} \quad \text { for all } k \geq 0,
$$

where $\|H\|_{k}$ denotes the least Lipschitz constant of $\mathrm{H}$ when the k-norm is used on the space $M(I, Y)$ and on the initial domain $D(\phi, Y) \subset M(I, Y)$.

Proof: Take any two functions $z_{1}$ and $z_{2}$ in $D(\phi, Y)$. Then from (2.5.1) we have the following estimate

$$
\begin{aligned}
\mid H\left(z_{1}\right)(t)- & H\left(z_{2}\right)(t)\left|\leq \int_{[0, t]}\right| z_{1}(s)-z_{2}(s) \mid d s \\
\leq & \int_{[0, t]}\left\|z_{1}-z_{2}\right\| k^{e^{k s}} d s \\
& \leq\left(\frac{1}{k}\right) e^{k t}\left\|z_{1}-z_{2}\right\|_{k}
\end{aligned}
$$

for almost all $t \in I$. Hence we get the inequality

$$
\left\|H\left(z_{1}\right)-H\left(z_{2}\right)\right\|_{k} \leq\left(\frac{1}{k}\right)\left\|z_{1}-z_{2}\right\|_{k}
$$


for any two functions $z_{1}$ and $z_{2}$ from $D(x, Y)$. This implies that $\|H\|_{k} \leq \frac{1}{k}$ for all $k>0$.

Previous Results: Assume that the operator $\mathrm{T}$ is nonanticipating Lipscitzian. The behavior of a dynamical system

$$
y(t)=f(t, y(t), T(y)(t)) \text { for almost all } \mathrm{t} \text { in } \mathrm{I}
$$

is known as an aftereffect differential equation if the operator $\mathrm{T}$ is a delay operator, with the initial domain $D(\phi, Y)$. The dynamical systems (3.1) is called a nonanticipating (or called hereditary system) operator differential equation. The existence and uniqueness of the solution to the dynamical system (1) has been investigated in [1] and [2].

The solution to a nonlinear differential equation in abstract space has been studied with different approaches during the past few decades (see Hale 1977 and Lashmikantham 1981). The existence and uniqueness of the solution to the generalized dynamical system of nonlinear operator differential equations has been proven by Bogdan (1981) and (1982).

The proof of existence and uniqueness of the solution to the nonlinear operator differential equation is presented in Bogdan (1981) and Ahangar 1986 and 1989.

$$
y(t)=\phi(t) \text { for almost all } \mathrm{t} \leq 0
$$

I) Solution of the System: Study the solution of the Nonlinear Operator Differential Equations (NODE)) or a dynamical system (3.2-a) together with the past history described by (3.2-b) is written in the following

$$
\left\{\begin{array}{c}
y \prime(t)=f(t, y(t), T(y)) \quad \text { for almost all } t>0 \\
y(t)=\phi(t) \quad \text { for a.a } \quad t \leq 0
\end{array}\right.
$$

where $\mathrm{f}$ and $\mathrm{T}$ have the same as above conditions. We will also investigate the solution to the perturbed system of the Equation (3)

$$
\left\{\begin{array}{c}
z^{\prime}(t)=f(t, z(t), T(z)(t))+g(t, z(t)) \quad \text { for a.a } t>0 \\
z(t)=\psi(t) \quad \text { for a.a } t \leq 0
\end{array}\right.
$$

The system (3.4) is said to the perturbation of the system (3.3) where $y(t)$ is the solution of the system (3.3).

II- Methodology and Procedure to be used: The results in Theorem 1, and Theorem 2, have been published. The method of functional analysis in Banach space and applied analysis can be used to prove.

III- Expected results and their Significance and/or Applications.

\section{Solution to the Perturbed Operator Dynamical System}

It would be interesting if we explore numerous applications or examples of this phenomena from mechanics, medicine, ecology, and automatic control that can be described by the operator differential equations. There are many examples in hyperbolic and parabolic PDE can be used when the nonanticipating operator is a delay operator. Computer algebra like Mathematica or Maple can be used to demonstrate the behavior of the solution to the nonlinear system. The operator $\mathrm{T}$ from the initial domain $D(\phi, Y) \subset M(I, Y)$ into the space of measurable functions $\mathrm{M}(\mathrm{I}, \mathrm{Z})$ is called nonanticipating (or causal) operator if the past information is independent from the future outputs.

Corollary 41: Let the operator $F$ in (2.4) and the operator $T$ in (3.1) be nonanticipating and Lipschitzian. The system of equation (3.3) has a unique solution $y \in D(\phi, Y)$.

A perturbation in the nonanticipating operator differential equation $y^{\prime}(t)=f(t, y(t), T(y)(t))$ will produce the following system

$$
z_{t}^{\prime}=f\left(t, z_{t}, T\left(z_{t}\right)\right)+g\left(t, z_{t}\right)
$$

$z_{t}^{\prime}=f\left(t, z_{t}, T\left(z_{t}\right)\right)+g\left(t, z_{t}\right)$

In a generalized dynamical system (3) and its perturbation (4) the function $\mathrm{f}$ is considered measurable in $\mathrm{t}$ and Lipschitzian in other variables. The result of this investigation will be valid in a special case functions $\mathrm{f}$ and $\mathrm{g}$ selected from the space of continuous functions in $Y=R^{n}$ that is $C(I \times Y, Y)$ and the interval $I=(0, a)$ or $(0, \infty)$.

Lemma 4.1: The Induced operator in (2.1) is Lipschitzian:

Proof: Let $f: I \times Y \times Z \rightarrow Z$ be a masurable in $\mathrm{t}$ and Lipschitzian with respect to other variables continuous function and

$$
\mid f\left(t, y, T(y)-f(t, z, T(z))\left|\leq L_{1}\right| y-z\left|+L_{2}\right| T(y)-T(z) \mid\right.
$$

for all $\mathrm{t}$ in the interval I. When the operator T is nonanticipating and Lipschitzian then by the relation (2) and (3)

$$
|T(y)-T(z)| \leq b|y-z| \leq b e^{k t}\|y-z\|_{k}
$$

The right hand side of the former inequality will be changed to 


$$
\begin{gathered}
\mid f\left(t, y, T(y)-f(t, z, T(z)) \mid \leq L_{1} e^{k t}\|y-z\|_{k}+L_{2} b e^{k t}\|y-z\|_{k}\right. \\
=\left(L_{1}+L_{2} b\right) e^{k t}\|y-z\|_{k} \\
|F(y)-F(z)| e^{-k t} \leq\left(L_{1}+L_{2} b\right)\|y-z\|_{k}
\end{gathered}
$$

Using the induced operator $\mathrm{F}$ and taking the ess.sup of the left hand side will obtain the following:

$$
\|F(y)-F(z)\|_{k} \leq L\|y-z\|_{k}
$$

where $L=L_{1}+b \cdot L_{2}$. In a special case when the transformation $T(y)=y$ is an identity operator then with the constant number $\mathrm{b}=1$ the Lipscitz contant number will be $L=L_{1}+L_{2}$. To apply this result to the perturbation function $g: I \times Y \rightarrow Z$ we consider a fixed perturbation function $g \in \operatorname{Lip}(I, Y ; Z)$ as an operator $H: D \subset M(I, Y) \rightarrow M(I, Z)$ such as

$$
z=H(y) \quad \text { if and only if } \quad z(t)=g(t, y(t))
$$

Similar argument can be applied to the H operator:

$$
\begin{gathered}
|H(y)-H(z)=| g(t, y)-g(t, z) \mid \leq l\|y-z\|_{k} \\
\|H(y)-H(z)\|_{k} \leq l\|y-z\|_{k}
\end{gathered}
$$

Theorem 4.2- (Integral Inequality): Let $\mathrm{y}(\mathrm{t})$ and $\phi(\mathrm{t})$ be two functions from the space $\mathrm{M}(\mathrm{I}, \mathrm{Y})$ and $c>0$. If

$$
|y(t)| \leq|\phi(t)|+c \int_{0}^{t}|y(s)| d s
$$

for almost all $t \in I$ then

$$
\|y\|_{k} \leq\|\phi\|_{k} \mid+\frac{c}{k}\|y\|_{k}
$$

for $k>0$ and almost all $t \in I$. For proof see Theorem 2, p.5, Bogdan 80FM21. Using the relation (1.2*) it can be concluded that

$$
|y(t)| \leq e^{k t}\left(\|\phi\|_{k} \mid+\frac{c}{k}\|y(s)\|_{k}\right)
$$

for $k>0$ and almost all $t \in I$.

Proof: To evaluate some estimate for the integral

$$
\int_{0}^{t}|y(s)| d s \leq \int_{0}^{t}\|y\|_{k} e^{k s} d s \leq\|y\|_{k} \frac{e^{k t}}{k}
$$

Use the inequality (4.5) and multiply both sides by $e^{-k t}$

$$
\begin{gathered}
|y(t)| \leq|\phi(t)|+c\|y\|_{k} \frac{e^{k t}}{k} \Rightarrow \\
|y(t)| e^{-k t} \leq|\phi(t)| e^{-k t}+c \frac{\|y\|_{k}}{k} \Longrightarrow
\end{gathered}
$$

for almost all $t$ in I. Take the ess.sup yields

$$
\|y\|_{k} \leq\|\phi\|_{k}+c \frac{\|y\|_{k}}{k}
$$

Theorem 4.3: Assume $f \in \operatorname{Lip}(I, Y ; Z), \mathrm{T}$ is a nonanticipating operator, and $(\phi, y) \in D(\phi, Y)$ be a solution to the operator differential equation (3.3), then

$$
\|y\|_{k} \leq\|\phi(t)\|_{k}+\frac{k\|f\|}{(k-\|f\|)}
$$

It is important to write the equivalent integral form of the system (3.3):

$$
y(t)=\phi(t)+\int_{0}^{t} f(s, y(s), T(y)(s)) d s
$$

Use the norm $|\mathrm{y}(\mathrm{t})|$ in $\mathrm{M}(\mathrm{I}, \mathrm{Y})$ to get the following inequality

$$
|y(t)| \leq|\phi(t)|+\int_{0}^{t}|f(s, y(s), T(y)(s))| d s
$$


Use the result from the relation (1.6) and substitute for the integrand $h(t)=f(t, y(t), T(y)(t))$

$$
\begin{aligned}
|y(t)| & \leq|\phi(t)|+\int_{0}^{t}|f(s, y(s), T(y)(s))| d s \\
& =|\phi(t)|+\int_{0}^{t}\|f\|(|y(s)|+1) d s .
\end{aligned}
$$

Use the previous result to substitute $|y(t)| \leq|\phi(t)|+c\|y\|_{k} \frac{e^{k t}}{k}$ will result the following

$$
\begin{aligned}
|y(t)| & \leq|\phi(t)|+\int_{0}^{t}\|f\|\left(|\phi(0)|+c\|y\|_{k} \frac{e^{k s}}{k}+1\right) d s \\
& \leq(|\phi(t)|+\|f\| t)+\|f\| \int_{0}^{t}|y(s)| d s
\end{aligned}
$$

Now use Theorem (2)

$$
\begin{gathered}
\|y\|_{k} \leq\|a\|_{k}+\frac{\|f\|}{k}\|y\|_{k} \\
a(t)=|\phi(t)|+\|f\| t \Longrightarrow\|a\|_{k} \leq\|\phi\|_{k}+\frac{\|f\|}{k} ? ? ? \\
\|y\|_{k} \leq\|\phi\|_{k}+\frac{\|f\|}{k}+\frac{\|f\|}{k}\|y\|_{k} \Longrightarrow \\
\|y\|_{k}\left(1-\frac{\|f\|}{k}\right) \leq\|\phi\|_{k}+\frac{\|f\|}{k} \Longrightarrow \\
\|y\|_{k} \leq\|\phi\|_{k}+\frac{k\|f\|}{(k-\|f\|)}
\end{gathered}
$$

Lemma 4.3: Let $\mathrm{f}$ be in the Lip - Space that is $f \in \operatorname{Lip}(I, Y ; Z)$ and $\mathrm{T}$ is nonanticipating and Lipscitzian operator. Assume that $\mathrm{y}$ is a solution to the unperturbed system (2.3) will satisfy the following relation

$$
\|y-z\|_{k} \leq b\|\phi-\psi\|_{k} \quad \text { for } b=\frac{k}{k-\|f\|\left(1+\|T\|_{k}\right)}
$$

where $\phi$ and $\psi$ are initial functions for $\mathrm{y}$ and $\mathrm{z}$ respectively.

Proof: The equation (2.1) is equivalent to the following integral form

$$
y(t)=\phi(t)+\int_{0}^{t} f(s, y(s), T(y)(s)) d s
$$

Assume both functions $\mathrm{y}(\mathrm{t})$ and $\mathrm{z}(\mathrm{t})$ are solutions to the system of (2.3) thus we will obtain the following estimate

$$
z(t)=\psi(t)+\int_{0}^{t}[f(s, z(s), T(z)(s)] d s
$$

for almost all $\mathrm{t}$ in I. Subtracting both sides of these equalities and using triangle inequality,

$$
|y(t)-z(t)| \leq|\phi(t)-\psi(t)|+\int_{0}^{t}[|f(s, y(s), T(y)(s))-f(s, z(s), T(z)(s))| d s
$$

Since $\mathrm{f}$ is Lipscitzian with respect to $\mathrm{y}$ and $\mathrm{T}$, using the Lipschitzian property the right hand side of this inequality will be $\left.\left.\leq|\phi(t)-\psi(t)|+\int_{0}^{t}\|f\|[|y(s)-z(s)|+\mid T(y)(s))-T(z)(s)\right)\right] \mid d s$ $\leq|\phi(t)-\psi(t)|+\int_{0}^{t}\|f\|\left[|y(s)-z(s)|+\|T\|_{k}|y(s)-z(s)|\right] d s$.

Using the inequality $(1.2 *)$

$\leq|\phi(t)-\psi(t)|+\int_{0}^{t}\|f\|\left[\|y-z\|_{k} e^{k s}+\|T\|_{k}\|y-z\|_{k} e^{k s}\right] d s$

$|y(t)-z(t)| \leq|\phi(t)-\psi(t)|+\|f\|\left[\|y-z\|_{k}\left[1+\|T\|_{k}\right] \int_{0}^{t} e^{k s} d s=\right.$

$|\phi(t)-\psi(t)|+\|f\|\left[\|y-z\|_{k}\left[1+\|T\|_{k}\right] \frac{1}{k}\left(e^{k t}-1\right)\right.$

$|\phi(t)-\psi(t)|+\|f\|\left[\|y-z\|_{k}\left[1+\|T\|_{k}\right] \frac{1}{k} e^{k t}\right.$

Multiply each side by $\mathrm{e}^{-k t}$

$|y(t)-z(t)| e^{-k t} \leq|\phi(t)-\psi(t)| e^{-k t}+\|f\|\left[\|y-z\|_{k}\left[1+\|T\|_{k}\right] \frac{1}{k}\right.$

Since t belongs to the real interval $I=[0, a]$ and by taking the ess.sup on the left hand side:

$\|y-z\|_{k}\left(1-\frac{\|f\|\left(1+\|T\|_{k}\right)}{k}\right) \leq\|\phi-\psi\|_{k}$

The final estimate for the variations on the solution will be Lipscitzian and 


$$
\|y-z\|_{k} \leq b\|\phi-\psi\|_{k}
$$

for $b=\frac{k}{k-\|f\|\left(1+\|T\|_{k}\right)}$ and $k \geq k_{0}=\|f\|\left(1+\|T\|_{k}\right)$.

Using the inequality $(1.2 *)$ this estimate can be interpreted to

$$
|y-z| \leq b e^{k t}\|\phi-\psi\|_{k}
$$

for $b=\frac{k}{k-\|f\|\left(1+\|T\|_{k}\right)}$.

Theorem (4.3): Assume functions $f \in \operatorname{Lip}(I, Y ; Z), g \in \operatorname{Lip}(I, Y)$, and the operator T is Nonanticipating and Lipscitzian. The perturbed operator $f \oplus g$ defined by

$$
h=(f \oplus g)(y) \Leftrightarrow h(t)=f(t, y(t))+g(t, y(t))
$$

is also nonanticipating and Lipschitzian.

\section{Perturbation in Operator Differential Equations}

Proposition 5.1: Given all conditions of the following Operator Differential Equation (2.8) and its perturbation (2.9), then the solution will satisfy the following inequality

$$
\|y-z\|_{k} \leq A\|\phi-\psi\|_{k}+B
$$

where $A=\frac{k}{\left.k-\left(\|f\|\left[1+\|T\|_{k}\right] \mid+\|g\|\right)\right)}$ and $B=\frac{\|g\|\left(\|\phi\|_{k}+\frac{k\|f\|}{(k-\|\| f \|}+1\right)}{\left(\|f\|\left[1+\|T\|_{k}\right] \mid+\|g\|\right.}$.

Proof: The equation (2.1) is equivalent to the following integral form

$$
\begin{gathered}
y(t)=\phi(t)+\int_{0}^{t} f(s, y(s), T(y)(s)) d s \\
y(t)=\phi(t)+\int_{0}^{t}[f(s, y(s), T(y)(s))+g(s, y(s))] d s-\int_{0}^{t} g(s, y(s)) d s
\end{gathered}
$$

Similarly, we write the equivalent integral form of the perturbed system (2.4)

$$
z(t)=y(t)+\int_{0}^{t}[f(s, z(s), T(z)(s))+g(s, z(s))] d s
$$

for almost all $\mathrm{t}$ in I. Subtracting both sides of these equalities and using triangle inequality and use Lemma (2)

$$
\begin{aligned}
|y(t)-z(t)| & \leq|\phi(t)-\psi(t)|+\int_{0}^{t}[|f(s, y(s), T(y)(s))-f(s, z(s), T(z)(s))|+ \\
& +|g(s, y(s))-g(s, z(s))|] d s+\int_{0}^{t}(\|g\|(|y(s)|+1) \mid d s
\end{aligned}
$$

Since $\mathrm{f}$ is Lipscitzian with respect to $\mathrm{y}$ and $\mathrm{T}$, by using definition, we can write

$$
\begin{aligned}
& \left.\left.\leq|\phi(t)-\psi(t)|+\int_{0}^{t}\|f\|[|y(s)-z(s)|+\mid T(y)(s))-T(z)(s)\right)\right] d s \mid \\
& \left.\left.\left.\left.+\|g\| \int_{0}^{t} \mid y(s)\right)-z(s)\right) \mid\right] d s+\|g\| \int_{0}^{t}(\mid y(s)) \mid+1\right) d s \\
& \leq|\phi(t)-\psi(t)|+\int_{0}^{t}\|f\|\left[|y(s)-z(s)|+\|T\|_{k}|y(s)-z(s)|\right] d s \mid \\
& +\|g\| \int_{0}^{t}\|y-z\|_{k} e^{k s} d s+\|g\| \int_{0}^{t}\left(\|y\|_{k} e^{k s}+e^{k s}\right) \mid d s \\
& \leq|\phi(t)-\psi(t)|+\int_{0}^{t}\|f\|\left[\|y-z\|_{k} e^{k s}+\|T\|_{k}\|y-z\|_{k} e^{k s}\right] d s \mid
\end{aligned}
$$

$$
+\|g\| \int_{0}^{t}\|y-z\|_{k} e^{k s} d s+\frac{1}{k}\|g\|\left(\|y\|_{k}+1\right) e^{k t}
$$

These estimates yield the following inequality

$$
\begin{gathered}
|y(t)-z(t)| \leq|\phi(t)-\psi(t)|+\|f\|\left[\|y-z\|_{k} \int_{0}^{t}\left[1+\|T\|_{k}\right] e^{k s} d s \mid\right. \\
+\|g\|\|y-z\|_{k} \int_{0}^{t} e^{k s} d s+\frac{\|g\|}{k}\left(\|y\|_{k}+1\right) e^{k t} . \\
\leq|\phi(t)-\psi(t)|+\|f\|\left[\|y-z\|_{k}\left[1+\|T\|_{k}\right] \frac{e^{k t}}{k}+\|g\|\|y-z\|_{k} \frac{e^{k t}}{k}\right. \\
+\frac{\|g\|}{k}\left(\|y\|_{k}+1\right) e^{k t} . \\
\leq|\phi(t)-\psi(t)|+\left(\|f\|\left[1+\|T\|_{k}\right] \frac{e^{k t}}{k}+\|g\|\right)\|y-z\|_{k} \frac{e^{k t}}{k}
\end{gathered}
$$




$$
+\frac{\|g\|}{k}\left(\|y\|_{k}+1\right) e^{k t}
$$

Multiply each side by $\mathrm{e}^{-k t}$

$|y(t)-z(t)| e^{-k t} \leq$

$|\phi(t)-\psi(t)| e^{-k t}+\left(\|f\|\left[1+\|T\|_{k}\right] \frac{1}{k}+\|g\|\right)\|y-z\|_{k} \frac{1}{k}+\frac{\|g\|}{k}\left(\|y\|_{k}+1\right)$

Since $\mathrm{t}$ belongs to the real interval $I=[0, a]$ and by taking the ess.sup on the left hand side:

$\|y-z\|_{k} \leq$

$\|\phi-\psi\|_{k}+\frac{1}{k}\left(\left(\|f\|\left[1+\|T\|_{k}\right] \mid+\|g\|\right)\|y-z\|_{k}+\frac{\|g\|}{k}\left(\|y\|_{k}+1\right)\right.$

Replace $\|y\|_{k}$ from the relation (2.11)

$\|y-z\|_{k} \leq$

$$
\|\phi-\psi\|_{k}+\frac{1}{k}\left(\left(\|f\|\left[1+\|T\|_{k}\right]+\|g\|\right)\|y-z\|_{k}+\frac{\|g\|}{k}\left(\|\phi\|_{k}+\frac{k\|f\|}{(k-\|f\|)}+1\right)\right.
$$

The k-norm term for $(\mathrm{y}-\mathrm{z})$ can be computed:

$\|y-z\|_{k}\left(1-\frac{1}{k}\left(\left(\|f\|\left[1+\|T\|_{k}\right] \mid+\|g\|\right)\right)\right.$

$\leq\|\phi-\psi\|_{k}+\frac{\|g\|}{k}\left(\|\phi\|_{k}+\frac{k\|f\|}{(k-\|f\|)}+1\right) \Longrightarrow$

$\|y-z\|_{k} \leq A\|\phi-\psi\|_{k}+B$ where

$A=\frac{k}{\left.k-\left(\|f\|\left[1+\|T\|_{k}\right] \mid+\|g\|\right)\right)}$ and

$B=\frac{\frac{\|g\|}{k}\left(\|\phi\|_{k}+\frac{k\|f\|}{(k-\|f\|)}+1\right)}{\frac{1}{k}\left(\left(\|f\|\left[1+\|T\|_{k}\right] \mid+\|g\|\right)\right.}=\frac{\|g\|\left(\|\phi\|_{k}+\frac{k\|f\|}{(k-\|f\|)}+1\right)}{\left(\|f\|\left[1+\|T\|_{k}\right] \mid+\|g\|\right.}$

$$
\|y-z\|_{k} \leq A\|\phi-\psi\|_{k}+B
$$

This proves the resultof the perturbation in Nonlinear Operator Differential Equations.

Corollary (5.1): Assume a sequence of pertubed function $\left\{g_{n}\right\} \in \operatorname{Lip}(I, Y)$ for the opertaor differential equations (3.3) such that $\lim _{n \rightarrow \infty} g_{n}=0$.

Let all conditions for the initial data, operator $\mathrm{T}$, functions $\mathrm{f}$ and $\mathrm{g}$ hold.

i) Then Limit of $\lim _{n \rightarrow \infty} B=0$ and

$$
\left\|y_{n}-z_{n}\right\|_{k} \leq A\left\|\phi_{n}-\psi_{n}\right\|_{k}
$$

ii) Assume $\left(\phi_{n}, y_{n}\right)$ is a solution sequence of (3.3) and $\left(\psi_{n}, z_{n}\right)$ is a solution sequence for (3.4). If $\mathrm{n} \rightarrow \infty$ then the limit solution $\left(\psi_{n}, z_{n}\right) \rightarrow\left(\phi_{n}, y_{n}\right)$.

\section{References}

[1] Ahangar R., "Existence of Optimal Controls for Generalized Dynamical Systems Satisfying Nonanticipating-Operator Differential Equations.” Dissertation, the Catholic University of America, Washington D.C 1986.

[2] Ahangar R., "Nonanticipating Dynamical Model and Optimal Control”, Applied Math Letter, vol. 2, No.1, pp. 15-18, 1989.

[3] and Salehi E., "Automatic Controls for Nonlinear Dynamical Systems with Lipschitzian Trajectories”, Journal of Mathematical Analysis and Applications 268, 400-405 (2002).

[4] Application of Functional Analysis to Perturbation Theory of Differential Equations. Johnson Space Center NASA, Publication 80-FM21 (1980), pp. 1-18

[5] Astolfi Alessandro, "New Results on the Global Stabilization of Minimum Phase Nonlinear Systems", Automatica vol.34, No. 6, pp. 783-788, 1998.

[6] Bogdan V.M., (1981), "Existence and Uniqueness of Solution for a class of Nonlinear Operator Differential Equations Arising in Automatic Spaceship Navigation,” NASA Technical Paper 1860, Springfield, VA, 1981.

[7] Bogdan V.M., "Existence and Uniqueness of Solution to Nonlinear Operator Differential Equations Generalizing Dynamical Systems of Automatic Spaceship Navigation,” Nonlinear Phenomena in Mathematical Sciences, Academic Press, pp. 123-136, 1982.99.

[8] Driver R. D., Ordinary and Delay Differential Equations”, Springer Verlag, 1977.

[9] Driver R. D. " Can the future influence the present?", Published for The American Physical Society by the American Institute of Physics 1978.

[10] Hale J., ”Theory of Functional Differential Equations”, Spring Verlag 1977. 
[11] Kerin M. G., Topics in Differential and Integral Equation and Operator Theory” ( Translated from Russian by Jacob A.), Berkhauser Verlag, 1983.

[12] Lakshmikantham, V. and Leela S., ”Nonlinear Differential Equations in Abstract Spaces”, Pergamon Press 1981.

[13] Rand R. H. and Dieter Armbruster, "Perturbation methods, bifurcation theory, and computer algebra”, New York, Spring Verlag, c1987.

[14] Steven H. Strogatz, "Nonlinear Dynamics and Chaos with applications to Physics, Biology, Chemistry, and Engineering", Addison Wesley, 1994.

[15] Shaked U., Yaesh I., and Souza C. E., "Bounded Real Criteria for Linear Time Delayed Systems", IEEE Transactions on Automatic Control, Vol. 43, No. 7, July 1998.

[16] Tutschke Wolfgang, "Solution of Initial Value Problems in Class of Generalized Analytical Functions", Springer Verlag 1989.

[17] Vainberg M. M., "Variational Method and Method of Monotone Operators, In the Theory of Nonlinear Equations", ( Translated from Russian by A. Libin and Edited by Louvish), John Wiley \& Sons 1973. 